\title{
Clinical Outcomes of Endoscope-Assisted 30-Gauge Single-Needle Technique for Intrascleral Intraocular Lens Fixation
}

\author{
Hayato Mitamura ${ }^{\text {a, }}$ Tatsushi Kaga ${ }^{a}$ Takashi Kojimac Norihiko Yoshidad \\ Hiroyuki Sato $^{\text {e Taisuke Matsuda }}{ }^{a}$ Sho Yokoyama ${ }^{a}$ Toshio Morib \\ Kazuo Ichikawa ${ }^{\mathrm{f}}$ \\ aDepartment of Ophthalmology, Japan Community Health Care Organization Chukyo Hospital, Nagoya, Japan; \\ ${ }^{b}$ Department of Ophthalmology, lida Municipal Hospital, lida, Japan; 'Department of Ophthalmology,

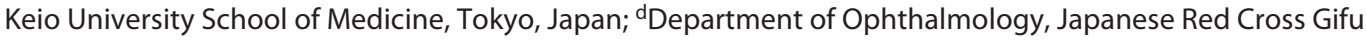

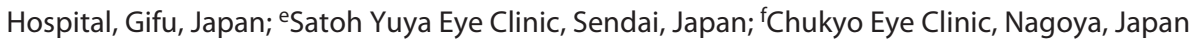

\section{Keywords}

Ophthalmic endoscopy · Ophthalmic surgery · Intrascleral intraocular lens fixation

\begin{abstract}
Introduction: We have developed an endoscope-assisted single-needle technique, which is an improvement of Yamane's double-needle technique of the intrascleral intraocular lens (IOL) fixation techniques. In this surgical procedure, the $\mathrm{IOL}$ is manipulated in the vitreous cavity, and the IOL haptic is externalized from the eye one by one with the aid of an ophthalmic endoscope. The purpose of this study was to report the postoperative visual function and safety of this new technique. Methods: Overall, 19 consecutive eyes (16 patients; mean age, $75.1 \pm 9.6$ years; mean follow-up period, 5.7 months) that underwent intrascleral IOL fixation surgery with our new technique were included in the study. Manifest refraction, uncorrected/corrected visual acuity, and corneal endothelial cell density were measured before and after surgery. Tilt and decentration of IOL were analyzed using anterior segment optical coherence tomography. Results: The mean absolute prediction error (spherical equivalent) was $0.82 \pm 0.52$. The mean postoperative best-corrected visual acuity had sig-
\end{abstract}

nificantly improved at the final visits $(p=0.02)$. No significant differences in the mean corneal endothelial cell density were observed between the first $\left(2,232 \pm 751\right.$ cells $\left./ \mathrm{mm}^{2}\right)$ and final $\left(2,099 \pm 649\right.$ cells $\left./ \mathrm{mm}^{2}\right)$ visits $(p=0.35)$. The mean IOL tilt was $8.1 \pm 3.2^{\circ}$. There were no vision-threatening complications, such as retinal detachment, endophthalmitis, or IOL dislocation, during or after surgery. Conclusions: The endoscopeassisted single-needle technique is a safe and effective method of intrascleral IOL fixation surgery.

(c) 2020 The Author(s)

Published by S. Karger AG, Basel

\section{Introduction}

Intrascleral intraocular lens (IOL) fixation is gaining popularity as a technique to insert an IOL in cases with insufficient capsule support [1,2], and several methods of performing this technique have already been reported [ $1-$ 8]. Among these, Yamane's double-needle method has proven superior so far as it reduces invasion to the conjunctiva and sclera relative to other techniques [1].

The key point of Yamane's double-needle technique is to insert and fix the leading haptic into the $30-\mathrm{G}$ needle, which compromises the mobility of the IOL optic and trail-
(C) 2020 The Author(s)

Published by S. Karger AG, Basel

This article is licensed under the Creative Commons Attribution 4.0 International License (CC BY) (http://www.karger.com/Services/ OpenAccessLicense). Usage, derivative works and distribution are permitted provided that proper credit is given to the author and the original publisher.
Takashi Kojima

Department of Ophthalmology

Keio University School of Medicine, 35 Shinanomachi

Shinjuku-ku Tokyo 160-8582 (Japan)

tkojkoj@mac.com 
ing haptic. Hence, the insertion of the trailing haptic into the $30-\mathrm{G}$ needle is difficult unless the position of the IOL insertion wound and the $30-\mathrm{G}$ needle puncture site is located $90^{\circ}$ apart. However, in actual clinical scenarios, gaining this position is challenging, especially in cases with narrow palpebral fissures or temporal IOL insertion wounds.

Therefore, we devised a new method in which IOL can be manipulated in the vitreous cavity, independent of the wound position. This method further improved visibility during the procedure in the vitreous cavity by employing an ophthalmic endoscope. To the best of our knowledge, this is the first study describing this new technique and reporting its clinical outcomes.

\section{Materials and Methods}

\section{Patients}

Patients who underwent intrascleral IOL fixation surgery between February 2017 and June 2018 at the Japan Community Health Care Organization, Chukyo Hospital and completed follow-up examination at 1 month after surgery were prescreened. Among them, data of consecutive cases using the endoscope-assisted single-needle technique were extracted, and finally, 19 eyes of 16 patients (female, 5; male, 11 ; mean age, $75.1 \pm 9.6$ ) were included in the study; these included 9 dislocated posterior chamber IOLs, 5 crystalline lens subluxations caused by zonular weakness that required simultaneous phacoemulsification and vitrectomy, and 5 aphakic eyes. The mean follow-up period was 5.7 months.

This retrospective study was approved by the Ethics Committee of the Japan Community Health Care Organization, Chukyo Hospital, and followed the tenets of the Declaration of Helsinki. The use of the opt-out method in place of obtaining informed consent was approved by the Ethics Committee.

\section{Endoscope-Assisted Single-Needle Technique}

Initially, a standard 23- or 25-G 3-port pars plana vitrectomy using the Constellation ${ }^{\circledR}$ Vision System (Alcon Laboratories, Inc., Fort Worth, TX, USA) or Fortas CV-30000 ${ }^{\circledR}$ (Nidek Co., Ltd., Aichi, Japan) was performed under sub-Tenon's anesthesia. Peripheral vitrectomy was performed without scleral compression under an endoscope. A 3-piece IOL (X70orNX70 [Santen, Osaka, Japan]) was used as the IOL for intrascleral fixation. The dislocated IOL was cut into 2 pieces and removed from the $3.2 \mathrm{~mm}$ corneal or sclerocorneal incision. The IOL injector was inserted into the eye, and the IOL was inserted until the leading haptic entered the vitreous cavity. The trailing haptic was left outside to prevent the IOL from falling into the vitreous cavity (Fig. 1a). A sclerotomy was performed through the conjunctiva using a 30-gauge thin-wall needle (TSK ultra-thin wall needle; Tochigi Seiko, Tochigi, Japan) $2 \mathrm{~mm}$ from the limbus. Under endoscopic observation, it was confirmed that the $30-G$ needle emerged at the ciliary sulcus (Fig. 1b). The IOL was further injected until the leading haptic and the optic were completely inserted into the vitreous cavity (Fig. 1c). The trailing haptic was gripped with forceps (Scharioth IOL scleral fixation forceps, DORC, Zuidland, Netherlands) and advanced into the vitreous cavity. The haptic was sub- sequently inserted into the $30-\mathrm{G}$ needle lumen under microscopy (Fig. 1d) and was externalized onto the conjunctiva by pulling the 30-G needle out of the sclera (Fig. 1e). A second sclerotomy was made with a $30-\mathrm{G}$ needle at a site situated $180^{\circ}$ from the first sclerotomy. The intraocular needle penetration site was confirmed with endoscopy (Fig. 1f). The other haptic was gripped with the forceps in the vitreous cavity under endoscopic observation (Fig. 1g). The haptic was moved to allow for its observation under the surgical microscope and was inserted into the $30-\mathrm{G}$ lumen (Fig. 1h). The externalized IOL haptics were cut with surgical microscissors to an appropriate length, and its ends were cauterized with an ophthalmic cautery device (Accu-Temp Cautery; Beaver Visitec, Waltham, MA, USA) and buried into the sclera over the conjunctiva. Before removing the 3 ports of pars plana vitrectomy, the fixed state of the IOL haptics and the intraocular condition was checked with an endoscope, and then the ports were removed. The supplementary explanatory video shows the endoscope-assisted 30 -gauge single-needle technique for intrascleral IOL fixation (see online suppl. video 1; for all online suppl. material, see www.karger.com/doi/10.1159/000511047).

\section{Measurement Items}

Manifest refractive sphere, cylinder, and spherical equivalent were evaluated before and after surgery. Distance uncorrected and corrected visual acuities were also measured using Landolt ring visual acuity chart. Decimal visual acuity was converted to the logarithm of the minimum angle of resolution ( $\log M A R)$.

The tilt and decentration of IOL were analyzed after surgery using the internal software of anterior segment OCT (CASIA2, TOMEY, Nagoya, Japan). Briefly, the perpendicular bisector of the line connecting the intersections of the front and back of the IOLs was defined as the "optical axis of the lens," while the center of the intersection was defined as "the center of the lens." Tilt was defined as the angle between the vertex normal and lens axis. Decentration was defined as the distance from the vertex normal to the lens center (Fig. 2). In tilt and decentration analysis, 3-dimensional analysis was performed based on two 2-dimensional orthogonal surfaces. A swept-source OCT biometer IOLMaster 700 (Carl Zeiss Meditec, Jena, Germany) was used for measurement of axial length and corneal curvature. The IOL calculation was performed using the SRK/T formula. A constant was optimized at our hospital, and the value 119.53 was applied.

\section{Statistical Analyses}

Comparison of parameters before and after surgery was conducted using the Wilcoxon signed-rank test. GraphPad Prism ver. 5.00 for Windows (GraphPad Software, San Diego, CA, USA) was used for all statistical analyses. A $p$ value $<5 \%$ was considered to be statistically significant.

\section{Results}

Table 1 shows the baseline characteristics of the 19 eyes of the 16 consecutive patients included in this study. Table 2 shows the summary of surgical outcomes. Among the patients, 9 and 10 patients involved superior and temporal wounds, respectively. 

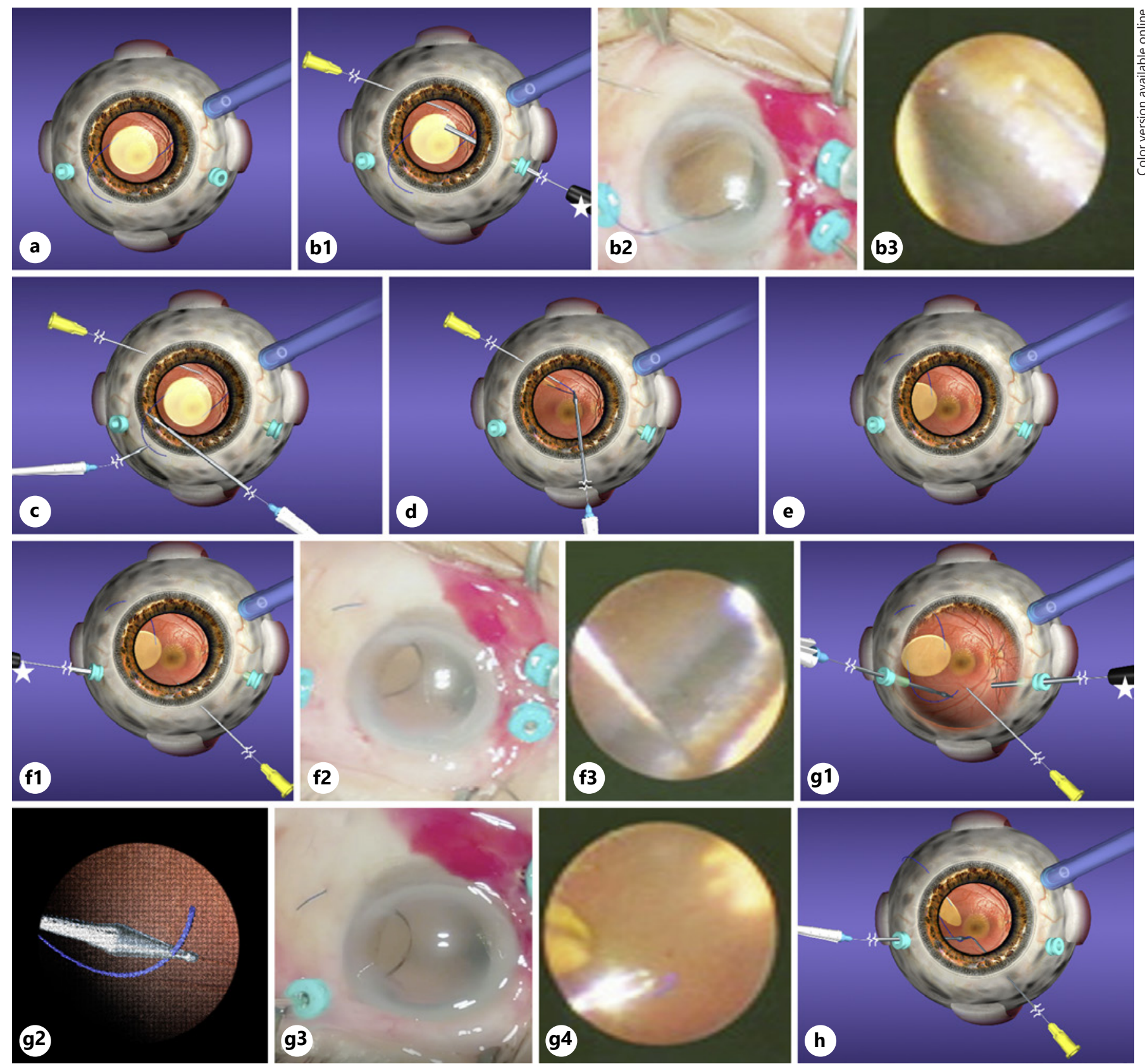

Fig. 1. Endoscope-assisted single 30-gauge needle technique for IOL intrascleral fixation. a The IOL is inserted into the anterior chamber and pushed forward until the leading haptic enters the vitreous cavity while the trailing haptic is kept outside the anterior chamber. Sclerotomy is created $2.0 \mathrm{~mm}$ from the limbus using a 30 -gauge needle (b1, b2), and the 30 -gauge puncture site is confirmed with an endoscope from the vitreous cavity side (b3). c The trailing haptics of the IOL are grasped with the IOL scleral fixation forceps from the corneal incision, and the whole IOL is moved into the vitreous cavity. The haptic is introduced into the lumen of the 30 -gauge needle in the vitreous cavity (d) and is externalized out of the sclera (e). The second sclerotomy is made $180^{\circ}$ apart from

the first sclerotomy $(\mathbf{f 1}, \mathbf{f 2})$, and the 30-gauge puncture site is confirmed with the endoscope from the vitreous cavity side (f3). g1-4 The other haptic is grasped with forceps with the aid of the endoscope in the vitreous cavity. $\mathbf{h}$ The haptic is moved to the position where it can be observed with the surgical microscope and is subsequently introduced into the 30 -gauge needle lumen. Created based on a video, a, b1, c, d, e, f1, g1, g2, h facilitate understanding of the technique. $\mathbf{b 2}, \mathbf{f} \mathbf{2}, \mathbf{g} \mathbf{3}$ are representative photographs obtained from a surgical video recorded during surgery with the single-needle technique. $\mathbf{b 3}, \mathbf{f 3}, \mathbf{g} \mathbf{4}$ are representative photographs obtained from a video recorded by ophthalmic endoscopy during the surgery with the single-needle technique. IOL, intraocular lens. 


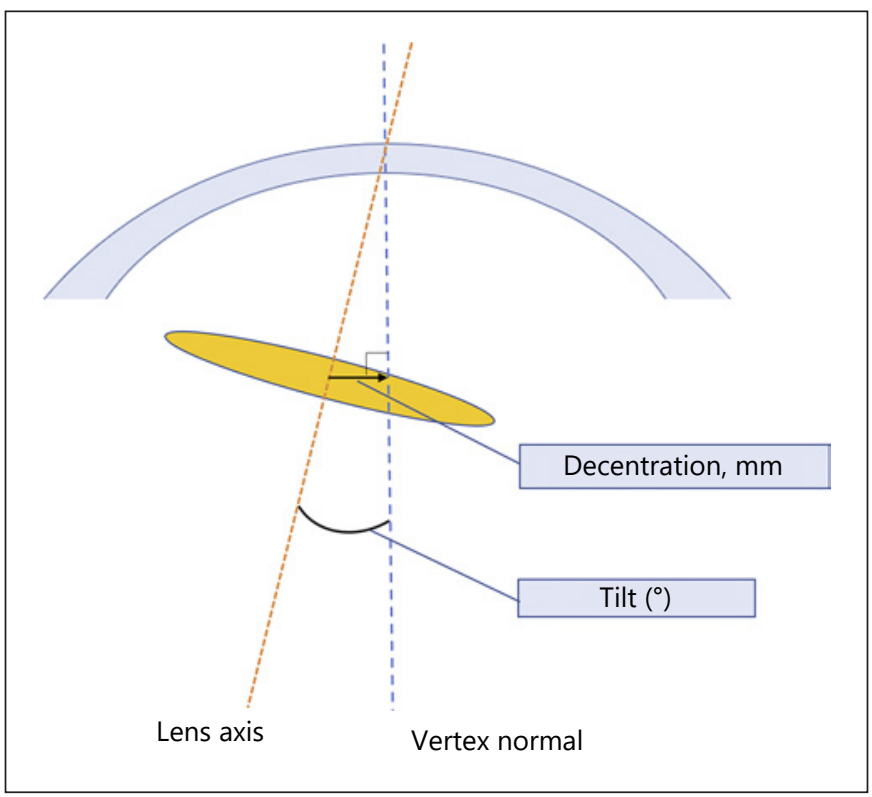

Fig. 2. Definition of IOL tilt and decentration. IOL tilt was defined as the angle between the vertex normal and IOL axis. Decentration was defined as the distance from the vertex normal to the lens center. IOL, intraocular lens.

\section{Visual and Refractive Outcomes}

The mean postoperative uncorrected visual acuity ( $\log$ MAR) in all patients was $0.57 \pm 0.40$. In patients whose refractive target was emmetropia and no other fundus disease, the mean uncorrected visual acuity was $0.39 \pm 0.33$. The mean postoperative corrected distance visual acuity (CDVA) $(0.013 \pm 0.14)$ significantly improved at the final visits $(p=0.02)$. There were no cases showing a decline of 2 or more lines in CDVA. The mean absolute prediction error of spherical equivalent was 0.82 $\pm 0.52 \mathrm{D}$.

\section{Corneal Endothelial Cell Density}

There were no significant differences in the mean corneal endothelial cell density between the first $(2,232 \pm 752$ cells $\left./ \mathrm{mm}^{2}\right)$ and final visits $\left(2,099 \pm 650\right.$ cells $\left./ \mathrm{mm}^{2}\right)(p=$ $0.35)$.

\section{Tilt and Decentration of IOL}

The mean IOL tilt was $8.1 \pm 3.2^{\circ}(3.5-13.8)$. The mean IOL decentration was $0.47 \pm 0.24 \mathrm{~mm}(1.1-0.14 \mathrm{~mm})$.

\section{Complications}

Table 3 shows that postoperative complications including IOL haptic exposure in 4 eyes $(21.0 \%)$, vitreous
Table 1. Patient demographic information

\begin{tabular}{ll} 
Characteristic & Value \\
\hline Number of eyes (patients) & $19(16)$ \\
Sex, male/female & $11 / 5$ \\
Mean age (range), years & $75(54-88)$ \\
Preexisting conditions, eyes, $n(\%)$ & \\
$\quad$ IOL luxation or dislocation & $9(47)$ \\
$\quad$ Aphakia & $5(26)$ \\
$\quad$ Zonular rupture during phacoemusification & $5(26)$ \\
Mean follow-up period after surgery (range), months & $5.7(3-15)$
\end{tabular}

IOL, intraocular lens; SD, standard deviation.

hemorrhage in 3 eyes (15.8\%), and hypotony in 1 eye (5.3\%). There were no incidents or complications, such as retinal detachment, endophthalmitis, or apparent IOL dislocation, that threatened postoperative vision. The cases of vitreous hemorrhage and hypotony resolved spontaneously. In the case of the exposed IOL haptic, the haptic was shortened, and the cauterized end was again buried in the sclera.

\section{Discussion}

Yamane's double-needle technique is an efficient, established technique for intrascleral IOL fixation. However, inserting the leading IOL haptic into the $30-\mathrm{G}$ needle can be challenging. When the IOL intrascleral fixation is initially planned, the IOL insertion wound position can be adjusted in consideration of the IOL $30-G$ needle puncture site. However, in actual clinical situations, obtaining the planned position is often difficult, especially in cases of complications during cataract surgery [9]. For example, in 1 case, a cataract surgery was scheduled with a temporal corneal incision, and in-the-bag IOL implantation was changed to intrascleral IOL fixation due to large zonular rupture during the surgery. In such cases, it is desirable to use the preexisting temporal corneal incision to perform the intrascleral IOL fixation surgery. Furthermore, in cases with a narrow palpebral fissure and deep-set eyes (Fig. 3a), it may be technically difficult to use a temporal IOL insertion wound because the eyelids interfere with the creation of the 30-G puncture site located at $90^{\circ}$ from the IOL insertion wound (Fig. 3b).

We, therefore, devised a single-needle technique: the whole IOL is guided into the vitreous cavity, and the trailing IOL haptic is inserted first into the $30-\mathrm{G}$ needle lumen 
Table 2. Visual acuity and corneal endothelial cell density before and after IOL intrascleral fixation

\begin{tabular}{lccc}
\hline & Before surgery & After surgery & $p$ value \\
\hline BCVA, logMAR & $0.30 \pm 0.48$ & 0.010 .14 & 0.02 \\
Corneal endothelial density, cells $/ \mathrm{mm}^{2}$ & $2,232 \pm 752$ & $2,099 \pm 650$ & 0.35 \\
\hline
\end{tabular}

Values are shown as the mean \pm SD. BCVA, best-corrected visual acuity; logMAR, logarithm of the minimum angle of resolution; SD, standard deviation; IOL, intraocular lens.

Table 3. Postoperative complications after IOL intrascleral fixation with the single-needle technique

\begin{tabular}{ll}
\hline Complication & Cases, $n(\%)$ \\
\hline Mild vitreous hemorrhage & $3(15.8)$ \\
IOL haptic exposure & $4(21.0)$ \\
Hypotony & $1(5.3)$ \\
\hline
\end{tabular}

IOL, intraocular lens.

and subsequently externalized out of the eye. The other haptic is then inserted into the $30-\mathrm{G}$ needle lumen and externalized out of the eye. Since the IOL can be moved freely in the vitreous space, this method allows the haptic to be inserted into the $30-\mathrm{G}$ needle lumen wherever the $30-G$ needle is located. Even in the cases where IOL intrascleral fixation is suddenly required or in cases featuring a narrow palpebral fissure and deep-set eyes, intrascleral IOL fixation can be safely performed regardless of the IOL insertion wound position (Fig. 3c-f). In this study, intrascleral IOL fixation surgery was successfully completed using superior IOL insertion wounds in 7 cases without any difficulty.

With this single-needle technique, the use of an endoscope helps to secure the outcome of the surgery. When one IOL haptic is externalized out of the sclera, the IOL optics and the other haptic are located under the externalized IOL haptic due to gravity and cannot be observed under a surgical microscope. This difficulty was overcome by employing an endoscopic observation in the vitreous cavity to find the IOL haptic and securing it with forceps.

In cases of poor mydriasis and those in which corneal edema has occurred due to intraoperative complications, a surgical microscope alone cannot gain sufficient intraocular visibility; this further complicates intrascleral IOL fixation with a $30-\mathrm{G}$ needle. However, by using an endoscope, our method improves visibility in the vitreous cav-

Endoscope-Assisted Technique for Intrascleral IOL Fixation ity without being influenced by corneal transparency and the pupil diameter. Even in such difficult cases, an endoscope thus allows for intrascleral IOL fixation to be performed with greater security.

Another advantage of using an endoscope during intrascleral IOL fixation is that the position of the $30-\mathrm{G}$ scleral puncture site can be directly confirmed. While a previous report describes the visualization of the needle penetration site with an endoscope during transscleral suture fixation [10], we observed the needle penetration with an endoscope during IOL intrascleral fixation in all cases. If the puncture site has greatly deviated from the ciliary sulcus, the $30-\mathrm{G}$ needle was re-punctured so as to permit its insertion into the vicinity of the ciliary sulcus. The endoscope also has been used for precise direct examination and trimming of the vitreous at the $30-\mathrm{G}$ scleral puncture site and peripheral retina, which may decrease the risk of postoperative retinal detachment [11].

In addition, since peripheral vitrectomy can be performed without scleral compression under endoscopic observation, postoperative inflammation may be reduced. In the future studies, intraocular inflammation should be compared between our new technique and the conventional double-needle technique.

At the end of the surgery, before removing the vitreous surgery ports, the endoscope was inserted from the port to check whether there was any vitreous strand around the IOL haptics and whether there was a retinal tear, so that the occurrence rate of postoperative retinal detachment may be prevented. In the future, it is necessary to compare the safety of this procedure with other procedures in a larger number of cases.

In this study, the postoperative changes of corneal endothelial cell density were comparable or superior to those described in previous reports [1, 3, 5-8]. Since our method involves the insertion of the IOL haptic into the $30-G$ needle in the vitreous cavity, it diminishes the risk of contact of the IOL or surgical instruments with the corneal endothelium relative to the standard double-needle 
Double needle technique

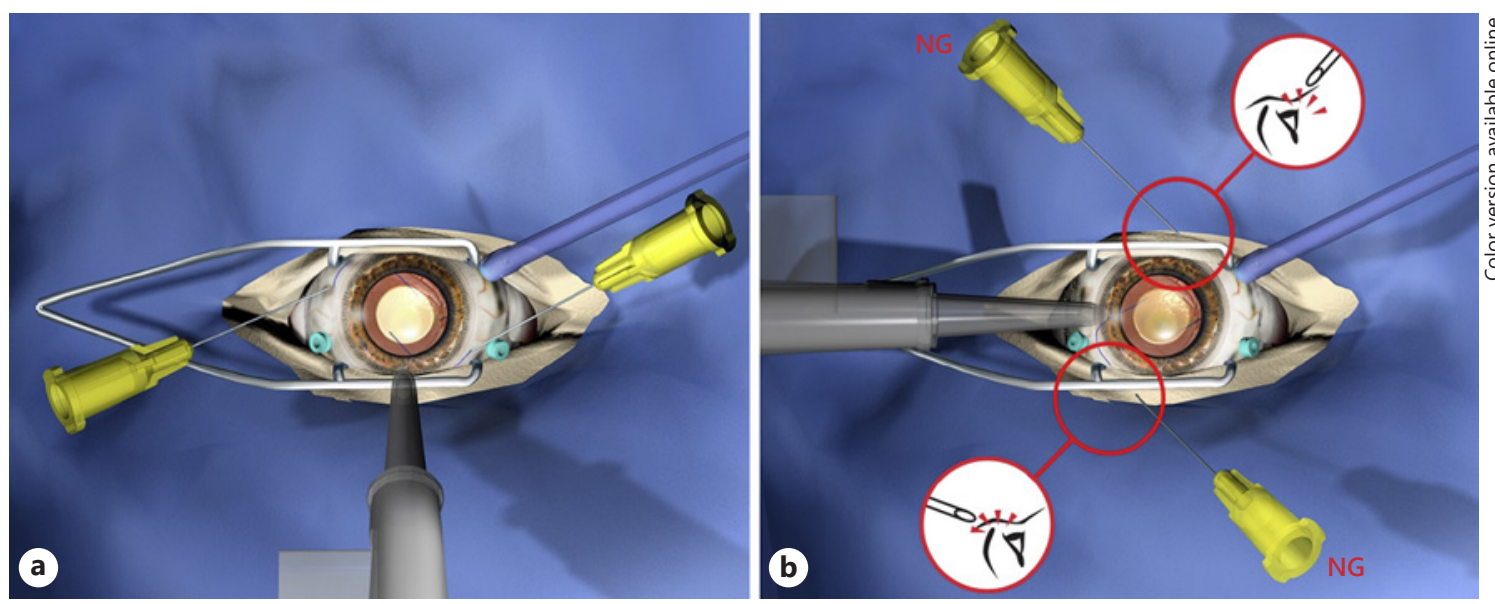

Single needle technique
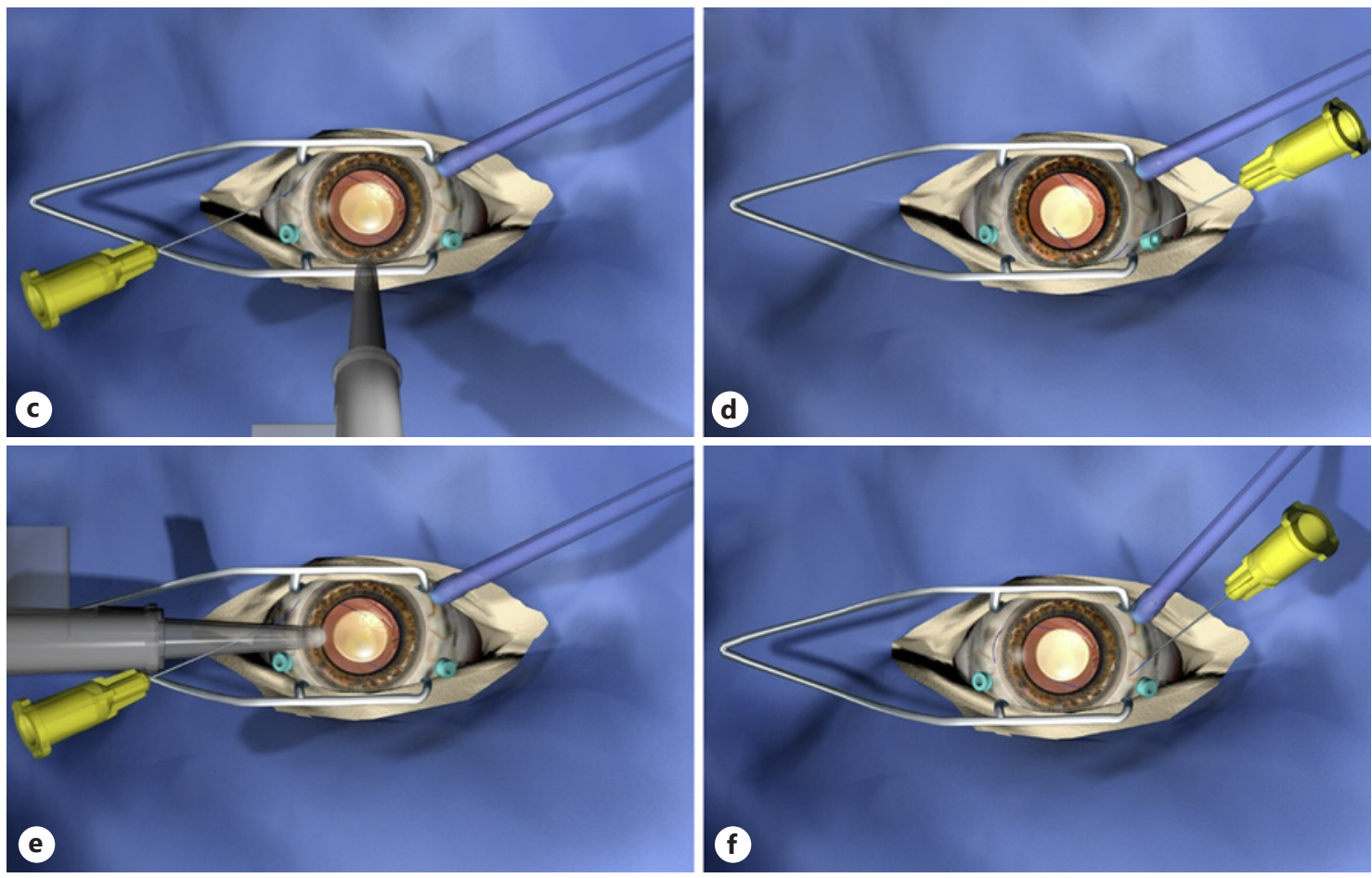

Fig. 3. IOL intrascleral fixation using the double-needle and singleneedle techniques in eyes with narrow palpebral fissure. a When the IOL is inserted from a superior wound, the Yamane's doubleneedle technique can be performed even in eyes with narrow palpebral fissures. b However, when the IOL is inserted from a temporal wound, creating the $30-\mathrm{G}$ sclerotomy at an appropriate position is obstructed by the eyelids. c When the IOL is inserted from

technique in which the main surgical procedure is performed in the anterior chamber. Further study with a larger number of cases and long-term follow-up is necessary to further validate these findings. Three cases showed the superior wound with our single-needle technique, the trailing haptic is first gripped with forceps and externalized from the eye. d The other haptic is then externalized from the eye. e Similarly, when the IOL is inserted into the eye from the temporal wound, the trailing haptic can be externalized from the eye. f Subsequently, the other haptic is externalized from the eye. IOL, intraocular lens.

postoperative IOL haptic exposure. As this could increase the risk of endophthalmitis and burden the patients with a foreign body sensation, the exposed part was cut, and the haptic end was cauterized and reburied under the 
conjunctiva. According to a previous report, this procedure is easy relative to the other treatments for exposed IOL haptics, such as a scleral patch graft [12]. However, further study is needed to evaluate the long-term safety and efficacy of this adjustment procedure.

Performing this single-needle technique risks the fall of IOL into the fundus. If the surgeon has insufficient experience with an ophthalmic endoscope, he or she can pick up the IOL under endoscopic observation. If not, it is recommended that a wide-angle viewing or contact lens system be used. Once the surgeon is accustomed to the use of an endoscope, he or she can grasp the IOL haptic under the endoscopic observation and resume the surgery.

Our current study found the mean IOL tilt to be $8.3^{\circ} \pm$ $4.3^{\circ}$, which was larger than a previously reported value $\left(3.4 \pm 2.5^{\circ}\right)[1]$. In the previous report, the tilt of the IOL was measured 2-dimensionally using an anterior segment OCT (SS-1000 CASIA; Tomey Corporation, Nagoya, Japan) [1]. By contrast, we measured the IOL tilt 3-dimensionally using an anterior segment OCT (CASIA2; Tomey Corporation, Nagoya, Japan). The mean IOL tilts cannot, therefore, be directly compared. However, the anterior segment OCT images revealed some cases to have featured an IOL tilt of relatively larger extent than those of conventional IOL implantation in the capsule. Future improvements of the technique will require the maintenance of the puncture angle of the $30-G$ needle at both ends to reduce the IOL tilt.

In addition, the present technique is limited by a required proficiency with the use of an endoscope. While all surgeries in this study were performed by vitreous surgeons skilled in endoscope-assisted vitrectomy, less technical proficiency in the skills required to perform the method herein proposed may affect the results. Further studies are needed to evaluate the effect of the skills of surgeons on the clinical outcomes in the future.

There are several limitations in this study. First, this study reported an early clinical outcome. The stability of IOL tilt and postoperative refraction should be evaluated with a long-term follow-up study. In addition, since the result of this study came from a single institution, potential bias by examiners and surgeons may be considered. Safety and efficacy should be examined in multicenter studies with a large number of cases in the future. There are many modifications in the intrascleral IOL fixation surgery. It is necessary to investigate in detail which technique can improve the prediction error of IOL power, IOL tilt, and surgically induced astigmatism. In conclusion, our newly devised single-needle technique with an ophthalmic endoscope is a safe, effective means of performing intrascleral IOL fixation even in eyes for which the procedure would otherwise prove challenging.

\section{Statement of Ethics}

This retrospective study was approved by the institutional review board of Japan Community Health Care Organization, Chukyo Hospital. The protocol of this study followed the tenets of the Declaration of Helsinki. The opt-out method was used in place of obtaining informed consent.

\section{Conflict of Interest Statement}

Dr. Kojima reports receiving personal fees from Alcon, Santen Pharmaceutical Co., Ltd., Otsuka Pharmaceutical, and TAAR Surgical outside the submitted work. Dr. Ichikawa reports receiving personal fees from Santen Pharmaceutical Co., Ltd., Alcon, and Kowa outside the submitted work.

\section{Funding Sources}

The authors did not receive any funding.

\section{Author Contributions}

All authors met the 4 conditions shown in the ICMJE criteria for authorship. Conception or design of the work: Mitamura H., Kaga T., Kojima T., and Ichikawa K. Data collection: Mitamura T., Yoshida N., and Sato H. Data analysis and interpretation: Mitamura H., Kojima T., Yokoyama S., and Mori T. Drafting of the article: Mitamura H. and Kojima T. Critical revision of the article: Kaga T., Kojima T., Yoshida N., Sato H., Matsuda T., Yokoyama S., and Ichikawa K. Final approval of the version to be published: Mitamura H., Kaga T., Kojima T., Yoshida N., Sato H., Matsuda T., Yokoyama S., and Ichikawa K.

\section{References}

1 Yamane S, Sato S, Maruyama-Inoue M, Kadonosono K. Flanged intrascleral intraocular lens fixation with double-needle technique. Ophthalmology. 2017 Aug;124(8):1136-42.

2 Ohta T, Toshida H, Murakami A. Simplified and safe method of sutureless intrascleral posterior chamber intraocular lens fixation: $y$ fixation technique. J Cataract Refract Surg. 2014 Jan;40(1):2-7.

3 Agarwal A, Kumar DA, Jacob S, Baid C, Agarwal A, Srinivasan S. Fibrin glue-assisted sutureless posterior chamber intraocular lens implantation in eyes with deficient posterior capsules. J Cataract Refract Surg. 2008 Sep; 34(9):1433-8. 
4 Totan Y, Karadag R. Trocar-assisted sutureless intrascleral posterior chamber foldable intra-ocular lens fixation. Eye. 2012 Jun; 26(6):788-91.

5 Gabor SG, Pavlidis MM. Sutureless intrascleral posterior chamber intraocular lens fixation. J Cataract Refract Surg. 2007 Nov; 33(11):1851-4.

6 Yamane S, Inoue M, Arakawa A, Kadonosono K. Sutureless 27-gauge needle-guided intrascleral intraocular lens implantation with lamellar scleral dissection. Ophthalmology. 2014 Jan;121(1):61-6.
7 Takayama K, Akimoto M, Taguchi H, Nakagawa S, Hiroi K. Transconjunctival sutureless intrascleral intraocular lens fixation using intrascleral tunnels guided with catheter and 30-gauge needles. Br J Ophthalmol. 2015 Nov; 99(11):1457-9.

8 Kawaji T, Sato T, Tanihara H. Sutureless intrascleral intraocular lens fixation with lamellar dissection of scleral tunnel. Clin Ophthalmol. 2016;10:227-31.

9 Kumar DA, Agarwal A, Packiyalakshmi S, Jacob S, Agarwal A. Complications and visual outcomes after glued foldable intraocular lens implantation in eyes with inadequate capsules. J Cataract Refract Surg. 2013 Aug;39(8): $1211-8$.
10 Sasahara M, Kiryu J, Yoshimura N. Endoscope-assisted transscleral suture fixation to reduce the incidence of intraocular lens dislocation. J Cataract Refract Surg. 2005 Sep; 31(9):1777-80.

11 El Gendy HA, Khalil HE, Haroun HE, El Deeb MW. Endoscopic-assisted scleral fixated IOL in the management of secondary aphakia in children. J Ophthalmol. 2016;2016:8501842.

12 Matsui Y, Matsubara H, Hanemoto T, Kondo $\mathrm{M}$. Exposure of haptic of posterior chamber intraocular lens after sutureless intrascleral fixation. BMC Ophthalmol. 2015 Dec;15:104. 